\title{
Flora representativa de las estribaciones occidentales de la cordillera en la provincia del Cotopaxi
}

\author{
Representative flora of the western foothills \\ of the mountain range in Cotopaxi Province
}

\author{
Marco Fernando Cerna Cevallos
}

Centro de Investigación y Valoración para la Biodiversidad CIVABI, Universidad Politécnica Salesiana, Quito, Ecuador.

* Autor para correspondencia: mcerna@ups.edu.ec.

Manuscrito recibido el 28 de noviembre de 2010.Aceptado, tras revisión el 25 de diciembre de 2010.

\begin{abstract}
Resumen
Esta investigación evalúa el estado de la flora en la zona occidental de la provincia del Cotopaxi; sobre la Estación 'Sacha Wiwa', como muestra de estudio, determinando la diversidad, estructura, composición de la vegetación, y características fenológicas de la flora. Se instaló una parcela permanente de una hectárea, tomando muestras de especies de más de $10 \mathrm{~cm}$ de DAP, además de colectar al azar muestras de herbáceas, epifitas, trepadoras fértiles y el registro de los datos fenológicos. Para complementar la información se añadieron las especies colectadas anteriormente por varios botánicos que visitaron el lugar. Se encontró 282 individuos correspondientes a 42 especies de árboles que tenían $10 \mathrm{~cm}$ ó más de DAP. La diversidad calculada de este bosque es baja; en toda el área se encontraron 202 especies en total, incluyendo hierbas, subarbustos, epifitas, arbustos, árboles y trepadoras. En los alrededores del lugar se identificó la presencia de 466 especies. Las especies más importantes en la estructura del bosque son: Wettinia equalis, Miconia brachycalyx y Aegiphyla alba. Las familias predominantes son: Arecaceae, Melastomataceae y Verbenaceae. Se encontraron 63 especies endémicas de las cuales 9 están 'en peligro', 10 están en la categoría 'casi amenazada', 20 son 'vulnerables'. Se describirán dos nuevas especies Anthurium sachawiwense y A. pucayacuense. La época con mayor número de especies fértiles va de octubre a enero. Se destaca la vegetación epifita de las familias Orchidiaceae, Araceae y Bromeliaceae por su número y endemismo. Así, se justifica la conservación del bosque y pueden planificarse actividades como la captura de $\mathrm{CO} 2$, el estableci-miento de un banco de semillas de especies nativas, producción y comercialización de especies ornamentales, el desarrollo de actividades científicas y turísticas.
\end{abstract}

Palabras clave: flora, fenología, bosque secundario tropical, endémico, Cotopaxi.

\begin{abstract}
This research evaluates the flora status in the western zone in Cotopaxi province, at "Sacha Wiwa" station as sample. Here the diversity, structure, composition of vegetation and phenological characteristics of the flora were determined.A hectare permanent parcel was installed in which samples the species whit more than $10 \mathrm{~cm} \mathrm{DBH}$ (diameter breast height) were marked and taken, besides samples of herbaceous plants, epiphytes, fertile creepers, and phenological data were collected randomly; 282 individuals corresponding to 42 species of trees that were $10 \mathrm{~cm}$ or more DBH; the calculated diversity if this forest is low with only 202 species including herbs, dwarf shrubs, epiphytes, shrubs trees and creepers; around the place 466 species were identified. The most important species in the structure of the forest are: Wettinia equalis, Miconia brachycalyx and Aegiphyla alba. The most predominant species are Arecaceae, Melastomataceae and Verbenaceae. In this place, 63 endemic species were found from which 9 are 'in danger,' 10 are in the 'almost threatened' category, 20 are 'vulnerable'. 2 new species will be described Anthurium sachawiwense and A. pucayacuense. Bigger number epoch of fertile species is from October to January. Referring to flora the epiphyte vegetation of the Orchidiaceae, Araceae and Bromeliaceae families stand out due to its number and endemism. Is justified the preservation of the forest and activities can be planned like the catch of $\mathrm{CO} 2$, the establishing of a seeds bank of native species, production and commercialization of ornamental species and the development of scientific and tourist activities.
\end{abstract}

Keywords: flora, phenology, tropical secondary forest, endemic, Cotopaxi.

Forma sugerida de citar:

Cerna, M. 20I0. Flora representativa de las estribaciones occidentales de la cordillera en la provincia del Cotopaxi. La Granja.Vol. I2(2). Pp 19-27. ISSN: I3903799. 


\section{Introducción}

La vegetación de las estribaciones de la cordillera de los Andes pertenece a un ecosistema que se ha mantenido en estado natural, por la geografía del terreno (ya que existe una gran cantidad de pendientes que lo conforman), factor que han determinado que el suelo no sea propicio para el desarrollo de actividades agrícolas; este factor a su vez ha permitido la formación de refugios de especies endémicas. Asimismo en el Ecuador existen zonas donde no se han realizado mayores estudios de las especies de flora, las que en muchos casos han desaparecido sin ser inventariadas o haber realizado colecciones botánicas de las diferentes especies (Palacios et al. y otros, 1997), esto debido a las actividades humanas, un ejemplo de ello se observa en los bosques naturales de los alrededores de los centros poblados como Coca, Lago Agrio y El Tena en el nororiente ecuatoriano. Esta situación nos plantea una importante y urgente tarea para los investigadores.

Con el fin de estudiar las características de la flora se estableció el método de las parcelas permanentes, esta metodología fue desarrollada por la Universidad de Oxford y es una herramienta de trabajo útil para evaluar los bosques tropicales (Brenes, 1991); las parcelas permanentes proveen datos (cuantitativos y cualitativos) sobre los cambios de la vegetación arbórea (Leaño y Saravia, 1998), este método ya ha sido utilizado en el Ecuador en casi todas las regiones.

Los estudios de la vegetación del Ecuador fueron iniciados hace 200 años. Ciertamente, se puede decir que por Alexander von Humbold; desde esta época varios botánicos han publicado descripciones de la vegetación y fitogeografía del Ecuador continental, estos investigadores serán descritos indicando el año de visita al país acontinuación: Sodiro (1874), Diels (1937), Acosta-Solís (1969, 1976), Harling (1979), Cañadas (I983), Jorgensen \& Yánez (1999), Entre los trabajos más sobresalientes.

Por otro lado, en la costa ecuatoriana pocos son los lugares estudiados de forma detenida, así: La Estación Científica 'Río Palenque' por Dodson y Gentry en 1979; la Estación Científica 'Pedro Franco Dávila' (Jauneche) por Dodson en 1985, Little y Dixon en 1969 hacen un estudio de los árboles de la provincia de Esmeraldas, La Cordillera de Chongón y Colonche fue estudiada por Valverde en I99I, El Parque Nacional Machalilla y la Reserva Ecológica 'Manglares Churute' en los cuales se realizó estudio de su flora para conocer su estado (Cerón, 1993).

De acuerdo con la base de datos 'Trópicos' del Missouri Botanical Garden (MO), El occidente de la provincia de Cotopaxi fue visitada por Thomas Croat, especialista en Araceae, en marzo de 1992; José Manzanares en junio de 199I, especialista en Bromeliaceae; Calagay Dodson especialista en Orchidaceae. Por conversaciones personales se conoce de la visita a esta zona de botánicos como: Carlos Cerón, Philip Silverston, H. Balslev, Efraín Freire, Germán Toasa, entre otros.

\section{I.I Bosque lluvioso de tierras bajas}

El bosque lluvioso de tierras bajas, de acuerdo a la clasificación de la vegetación Harling, ésta cubre el norte de las tierras bajas de la costa del Pacífico bajo los 700 msnm de elevación, la mayor parte de Esmeraldas, las áreas adyacentes de la provincia de Pichincha y áreas pequeñas del norte de Manabí y los Ríos.

El bosque lluvioso de tierras bajas se caracteriza por un clima con una precipitación anual sobre los $3.000 \mathrm{~mm}$ y carece de una estación seca marcada. La vegetación es densa y siempre verde, con el dosel frecuentemente de 30 metros ó más de altitud y una diversidad alta de especies. La diversidad alfa de los árboles, como se muestra en las parcelas permanentes de una hectárea, es más alta en la Amazonía ecuatoriana que en él arrea del bosque lluvioso en el norte de la costa del Pacífico, así en la Amazonía se encuentran de 200 a 240 especies de árboles de más de $10 \mathrm{~cm}$ de DAP, mientras que en la costa se han encontrado hasta IIO y 120 especies.

El bosque lluvioso en las tierras bajas del noreste del Ecuador es muy similar al de la región del Chocó colombiano de la costa del Pacífico y comparte muchas especies, pero hay también un elemento significativo de especies endémicas que no se conocen al norte de la frontera colombiana (Jorgensen \& León-Yánez, 1999).

\section{I.2 Estratos del bosque}

En la selva se da una competición de especies por la luz del sol, se presentan especies umbrófilas aficionadas a la penumbra y especies heliófilas, amigas de la luz.

En este proceso de ubicación y ordenamiento, todas las especies se localizan en estratos claramente diferenciados que son desde el más bajo y oscuro hasta él más alto y claro: rastrero, herbáceo, arbustivo, arborescente y emergente.

Según otra clasificación los estratos de la selva son 3: sotobosque (0-2,5 m) el dosel formado por el estrato arbóreo $(2,5-30 \mathrm{~m})$ y el dosel superior $(30-50 \mathrm{~m})$ formado por el estrato emergente que agrupa a todos los gigantes de copas umbelíferas que salen de la línea de cobertura convencional varios metros hacia arriba.

\section{I.3 Consecuencias de la destrucción del bosque tropical}


Entre los principales daños tenemos: daños a los complejos hidroeléctricos, la obstaculización de las vías de navegación, la desestabilización climática, la erosión, la pérdida de vida silvestre y de madera preciosa.

La eliminación de la cobertura vegetal hace que disminuya la infiltración de las aguas. El arrastre de sedimentos produce una disminución del periodo de vida útil de los complejos hidroeléctricos (Bonilla, 1983).

\section{I.4 La vegetación epifita}

Las epifitas tienen una particular importancia en el bosque, ya que constituyen el refugio de muchas especies, además de que producen gran parte de los recursos alimenticios.

Las especies de epifitas más importantes son Orchidaceae, Araceae, Bromeliaceae, Ericaceae y el grupo de los Helechos.

Las epifitas vasculares pueden ser utilizadas como indicadores biológicos de alteración de ambientes, ya que algunas especies de aráceas y helechos epifitos podrían encontrarse restringidos a bosques no disturbados (Larrea, 1997).

\section{Materiales y métodos}

\section{I Área de estudio}

El estudio se realizó en el Centro de Investigaciones 'Sacha Wiwa' que se encuentra en las estribaciones occidentales de la cordillera de los Andes; políticamente se ubica en la parroquia Guasaganda del cantón La Maná de la provincia de Cotopaxi. Geográficamente se encuentra en las coordenadas $0^{\circ} 47^{\circ} \mathrm{S} 79^{\circ}$ $09^{\circ} \mathrm{W}$, a una altura de $550 \mathrm{msnm}$ (Figura I). En este Centro de Investigaciones se encontró vegetación representativa de una zona de vida de aproximadamente 30.000 ha que se encuentra junto a las poblaciones de Pucayacu, Guasaganda y El Guayacán. La formación natural corresponde a un bosque húmedo tropical pie montano de la costa del Ecuador (Cerón, 1999). Según los datos del Instituto Nacional de Meteorología e Hidrología - INAMHI, la zona tiene una temperatura media anual de $24^{\circ} \mathrm{C}$, humedad relativa del $92 \%$, y una precipitación anual promedio de $2.828 \mathrm{~mm}$. Presenta una época lluviosa entre diciembre y mayo con un pico en febrero y marzo, un periodo de disminución de la lluvia entre junio y noviembre (INAMHI, 1998).

\subsection{Población y muestra}

Se procedió a fijar los límites de la zona de estudio, estableciendo una franja en las estribaciones occidentales de la cordillera de los Andes en la provincia de Cotopaxi entre los 400 y 700 msnm en los alrededores de las poblaciones de El Guayacán, Guasaganda y Pucayacu; esta franja posee características geográficas similares y tiene una área aproximada de 30.000 ha de las cuales la Estación 'Sacha Wiwa' tiene II 5 hectáreas. Para la realización del estudio se demarcó una muestra de una hectárea en

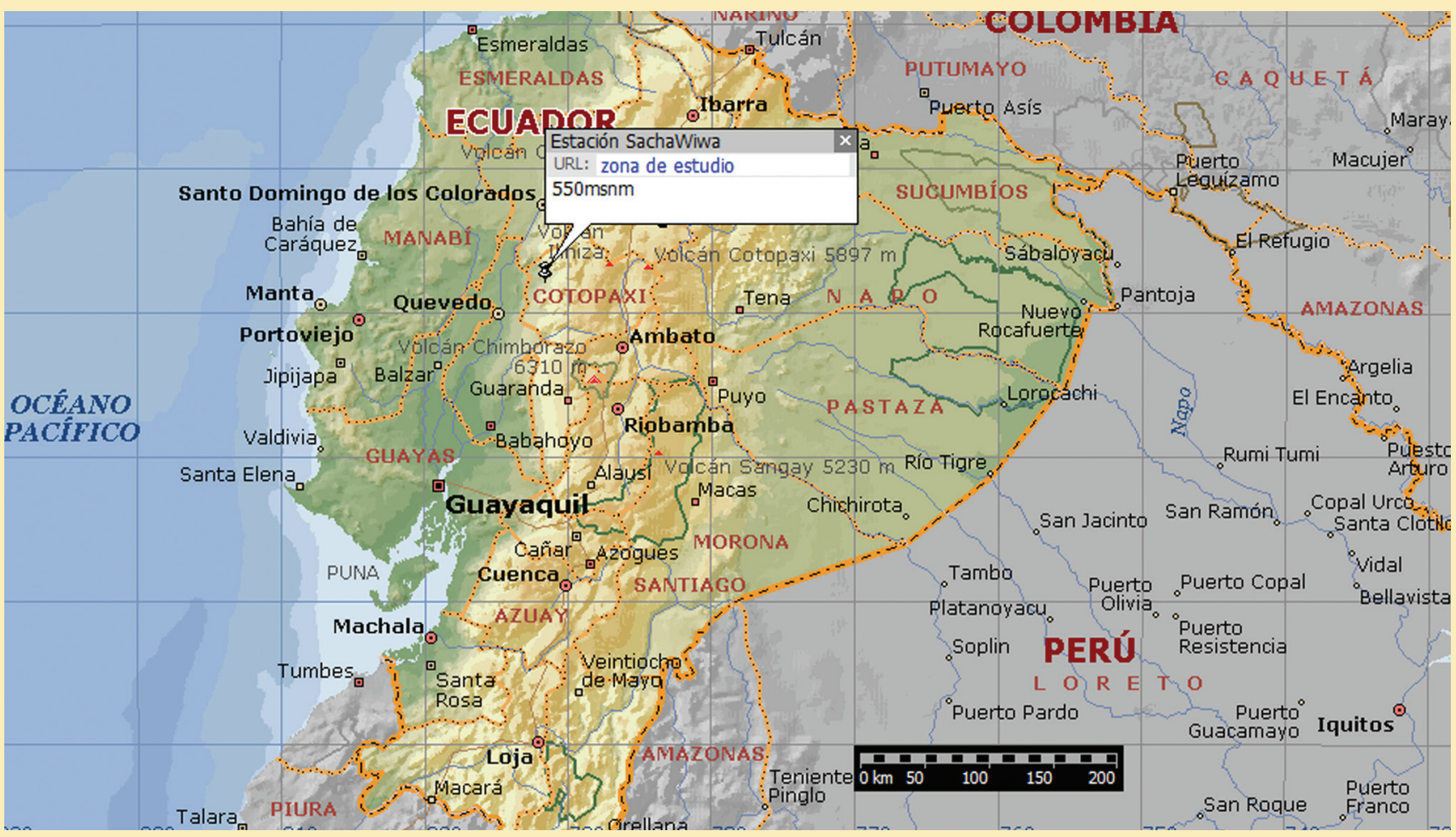

Figura 1. Ubicación de la Estación ‘Sacha Wiwa’ en el Ecuador, Fuente Encarta, 2007. 
donde la población y las muestras son:

Población: Vegetación existente en 30.000 hectáreas. Muestra I: Vegetación existente en II 5 hectáreas. Muestra 2: Vegetación existente en I hectáreas.

\subsection{Técnicas e instrumentos de la investigación}

Para este trabajo se desarrolló primero una etapa de exploración en 5 transectos de $100 \mathrm{~m} \times 4 \mathrm{~m}$ distribuidos transversalmente a todo lo largo del bosque que se encuentra en la Estación 'Sacha Wiwa'; luego de lo cual se identificó el lugar más adecuado para instalar una parcela permanente de $100 \mathrm{~m} \times 100 \mathrm{~m}$ (I hectárea); en los transectos $y$ en la parcela se colectaron muestras de cada especie $\geq$ a $10 \mathrm{~cm}$ de DAP (Diámetro a la Altura del Pecho a I,5 $\mathrm{m}$ de altura) y las especies fértiles de herbáceas epifitas y trepadoras encontradas en los alrededores de la zona de estudio; se registró además datos fenológicos ocasionales (floración, fructificación e infertilidad) Ver Figura 2.

La identificación se realizó en el Herbario $\mathrm{Na}$ cional del Ecuador (QCNE). Los duplicados de las muestras fueron entregados en el Herbario Alfredo Paredes QAP, en el Herbario Nacional del Ecuador QCNE y en el Herbario de la Universidad Politécnica Salesiana de Quito.

\subsection{Técnicas de análisis de datos}

Se realizaron los siguientes análisis: área basal, densidad relativa, frecuencia, frecuencia relativa, dominancia relativa, Valor de importancia por especie género y por familia. Además de la diversidad mediante el índice de

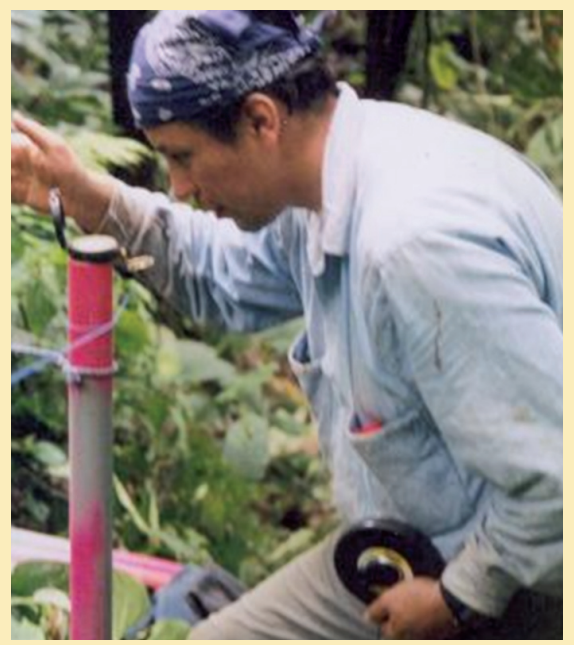

Alineación de la parcela permanente

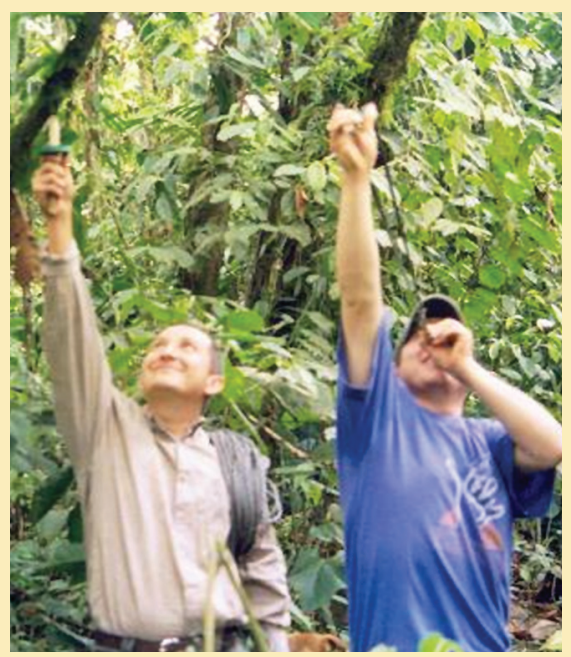

Colección de muestras

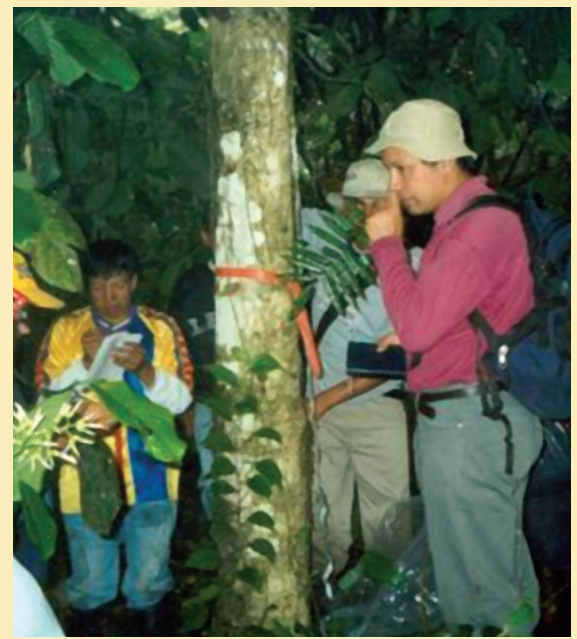

Colección de muestras

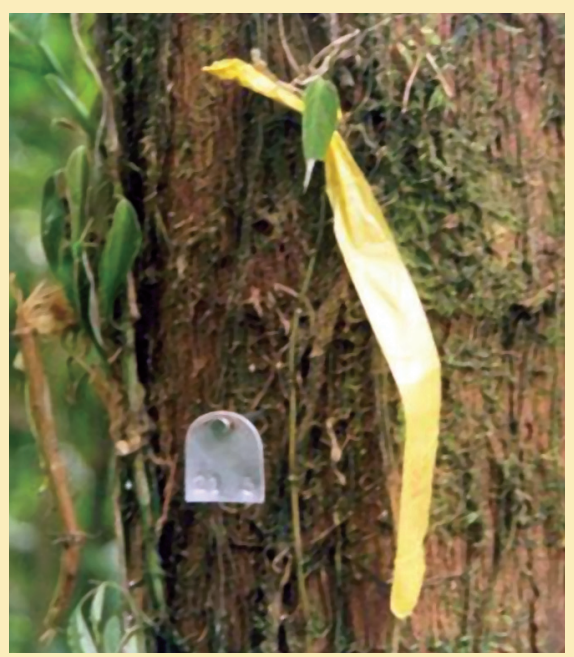

Marcaje de árboles con cinta y placas de aluminio

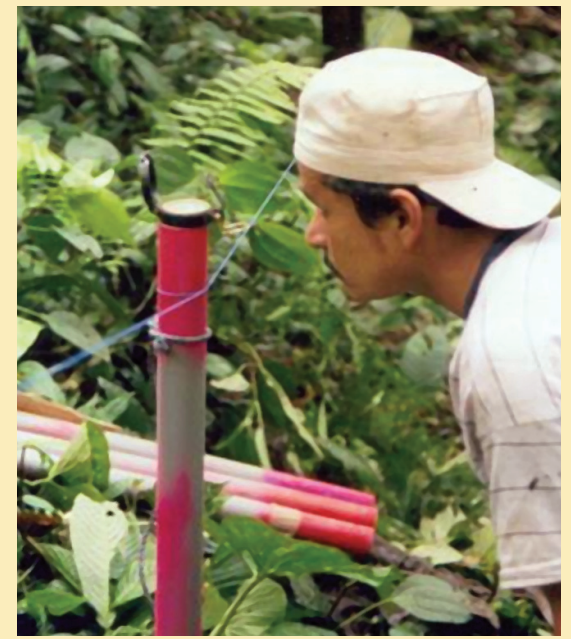

Establecimiento de subparcelas

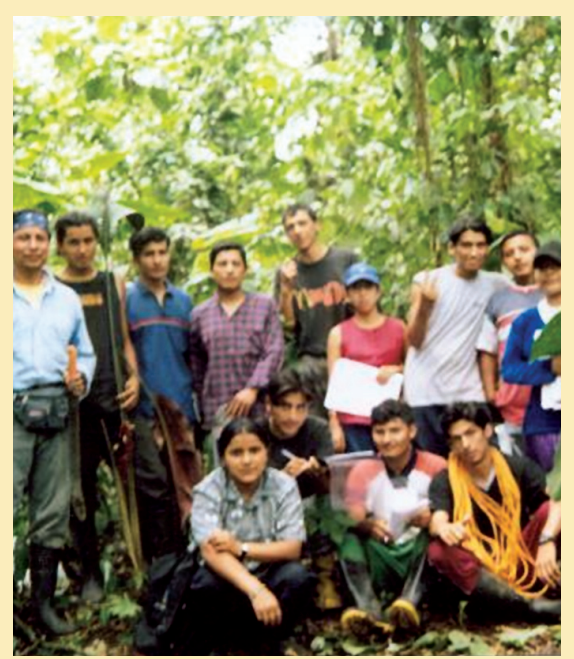

Establecimiento de subparcelas

Figura 2. Trabajos de campo para la investigación de la flora de la Estación 'Sacha Wiwa'. 
Simpson; usando los datos tomados en el campo y las identificaciones de herbario. Se tomaron en cuenta las indicaciones de (Cerón, 1993; Uslar et al., 2003).

\subsection{Estratificación de las poblaciones vegetales}

Para este fin se recopiló la información de las especies colectadas en la zona de estudio por varios botánicos, registrados en la Base de datos Trópicos del Missouri Botanical Garden y las especies encontradas en el presente estudio; las especies encontradas se las dividió en estratos de acuerdo con su forma de vida o lugar que ocupan en el bosque; los estratos formados fueron: Herbáceas, Subarbustos, Arbustos, Trepadora, Hemiepífita, Epifitos, Árboles.

\subsection{Fenología ocasional de las especies vegetales}

Para cada especie colectada se determinó un calendario anual en el cual se estableció una época aproximada de floración y fructificación; para esto se tomaron los datos del estado fenológico de cada especie en el momento de su colección y en las observaciones hechas en la parcela permanente en diferentes épocas del año.

En cada mes se determinó el número de especies que estaban en floración o fructificación con el fin de determinar en que época del año existe mayor fertilidad en el bosque. Estos datos se compararon con los registros de lluvia emitidos por la estación metereológica 'San Juan' de La Maná perteneciente al INAMHI; ubicada a 15 km de la Estación 'Sacha Wiwa', con el propósito de entender la relación entre la fertilidad y la cantidad de lluvias.

\subsection{Especies endémicas y/o en peligro de}

\begin{tabular}{cc}
\hline Aegiphyla alba & 38,99 \\
Aiconia brevitheca & 33,44 \\
Cecropia marginalis & 11,54 \\
Carapa megistocarpa & 11,30 \\
Inga oerstediana & 11,29 \\
Dussia lehmannii & 8,70 \\
Licania macrocarpa & 5,69 \\
Eschweilera rimbachii & 5,64 \\
Vismia lauriformis & 5,64 \\
Ficus macbridei & 5,17 \\
PROMEDIO &
\end{tabular}

Figura 3. Índice de Valor de Importancia de las especies iguales o mayores a $10 \mathrm{~cm}$ de DAP de la parcela permanente en la Estación 'Sacha Wiwa'.

\section{extinción}

Las especies registradas para la zona fueron comparadas con las listas del Libro Rojo de las Especies Endémicas del Ecuador 2000; estas fueron ubicadas dentro de las categorías de la Unión Internacional para la Conservación de la Naturaleza y de los Recursos Naturales (UICN): 'Extinto' (EX), 'Extinto en la naturaleza' (EW), 'En Peligro Crítico' (CR), 'En Peligro' (EN), 'Vulnerable” (VU), 'Casi Amenazado' (NT), 'Preocupación Menor' (LC), 'Datos Insuficientes' (DD), 'No Evaluado' (NE).

Nota. Se incluyó otra categoría no registrada en los parámetros de la UICN 'No Amenazada' (NA) para utilizarla con las especies Nativas e Introducidas que tienen una amplia distribución.

\section{Resultados}

En una hectárea de bosque secundario y disturbado en la Estación 'Sacha Wiwa' se encontró 282 individuos correspondientes a 42 especies de árboles que tenían $10 \mathrm{~cm}$ ó más de Diámetro a la Altura del Pecho (DAP). Estas especies pertenecen a 35 géneros de 25 familias. Los taxones más importantes en la estructura del bosque son: Wettinia equalis, Miconia brachycalyx y Aegiphyla alba (Figura 3) que son las especie más abundantes, tienen mayor área basal y además tienen el mayor Índice de Valor de Importancia. Las familias más importantes en la estructura del bosque son Arecaceae y Melastomataceae.

$\begin{array}{cc}\text { ESPECIE } & \text { I.V.I. } \\ \text { Miconia brachycalyx } & 54,92 \\ \text { Wettinia equalis } & 47,77\end{array}$

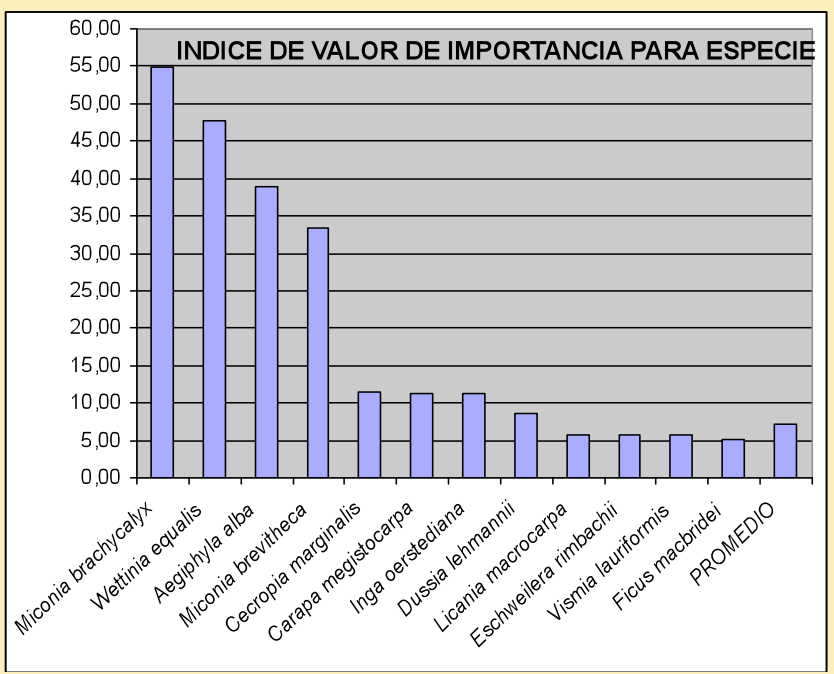


En la hectárea de bosque secundario en estudio se encontró $16.368 \mathrm{~m}^{2}$ de área basal total, este valor demuestra poca importancia para la explotación maderera.

El Bosque de la Estación 'Sacha Wiwa' tiene un índice de diversidad calculado de 7,63; lo que nos indica que el bosque se ubica en el nivel de diversidad baja. Esto se debe a que se trata de un bosque secundario en recuperación y está poblado en su mayoría por especies de ciclos de vida cortos como hierbas, arbustos, trepadoras y epĺfitas. Este bosque se encuentra en recuperación y tiene una baja diversidad en especies forestales pero encontramos especies hospederas de epifitas pertenecientes a las familias Orchidaceae,Araceae y Bomeliaceae en estos grupos existen especies endémicas en peligro o poco estudiadas.

En esta área se registraron 202 especies en total incluyendo hierbas, subarbustos, epifitas, arbustos, árboles y trepadoras; estas especies pertenecen a 147 géneros que están incluidos en 78 familias botánicas. En el estrato bajo existen 86 especies en el estrato medio hay 13 especies y en el estrato alto 103 especies.

En este lugar se encontraron 20 especies endémicas de las cuales 2 están en la categoría 'En Peligro' Herrania balaensis (Sterculiaceae) y Podandrogyne brevipedunculata (Capparaceae); 2 están en la categoría 'Vulnerables'; 2 están ‘Casi Amenazadas'.

\begin{tabular}{lccc}
\hline Origen & $\mathrm{N}^{\circ}$ & Estado de conservación & $\mathrm{N}^{\circ}$ \\
\hline endémica & 20 & En peligro & 2 \\
& & Vulnerable & 2 \\
& & Casi amenazado & 2 \\
& & Datos insuficientes & II \\
& & & 3 \\
\hline introducida & 28 & No amenazada & 182 \\
\hline nativa & 154 & & total \\
& & & 202 \\
\hline
\end{tabular}

Los meses en los cuales existe mayor número de especies fértiles van desde octubre hasta enero, pero el mayor pico se encuentra en noviembre. La época con menos especies fértiles está entre marzo y agosto. Al comparar los datos de los meses de mayor número de especies fértiles con los datos del promedio de lluvias mensuales (Figura 5), se encontró que los picos de fertilidad coinciden con el inicio de la época lluviosa en septiembre, y la época menos fértil está entre mayo y agosto que es una temporada de pocas lluvias (Figuras 6 y 7$)$.

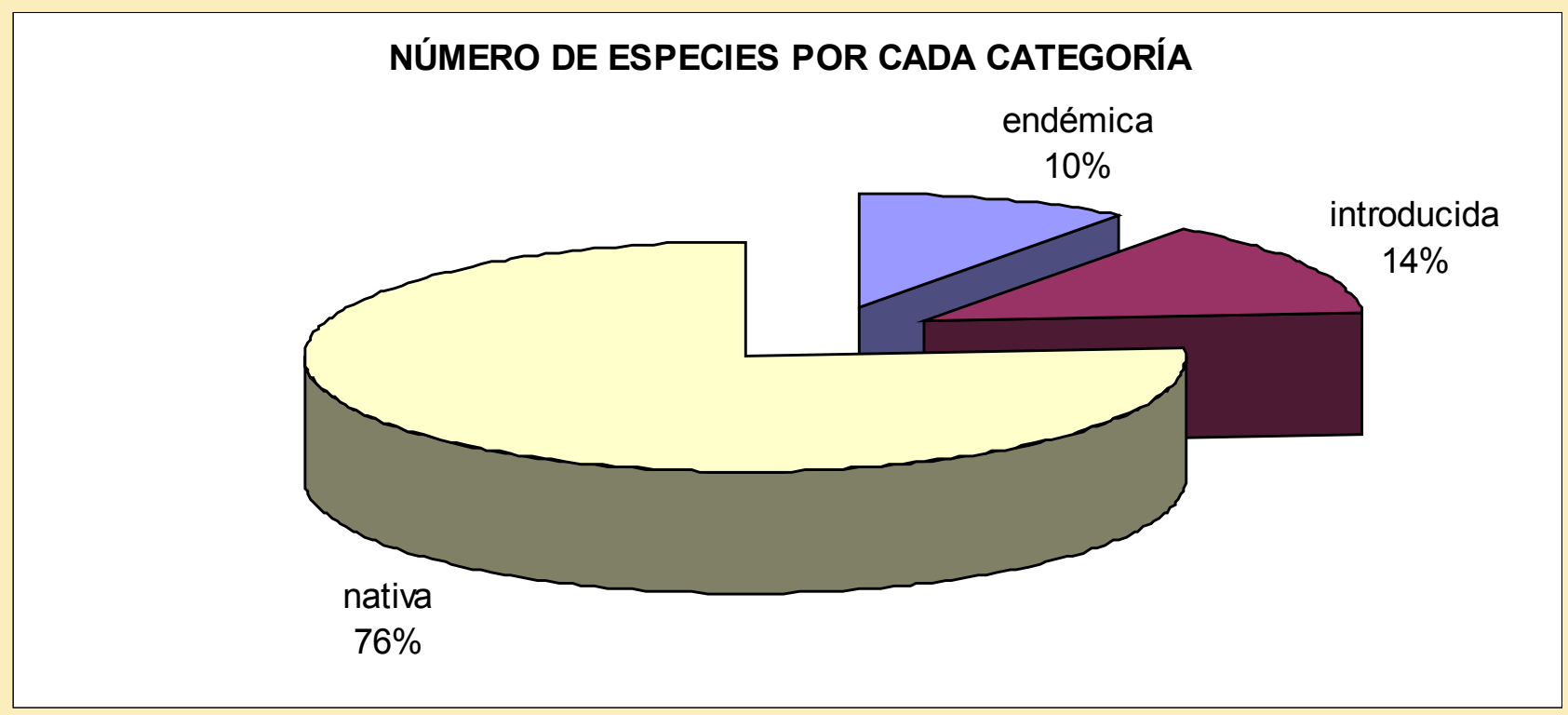

Figura 4. Categorías de origen de las especies en la Estación 'Sacha Wiwa'. 


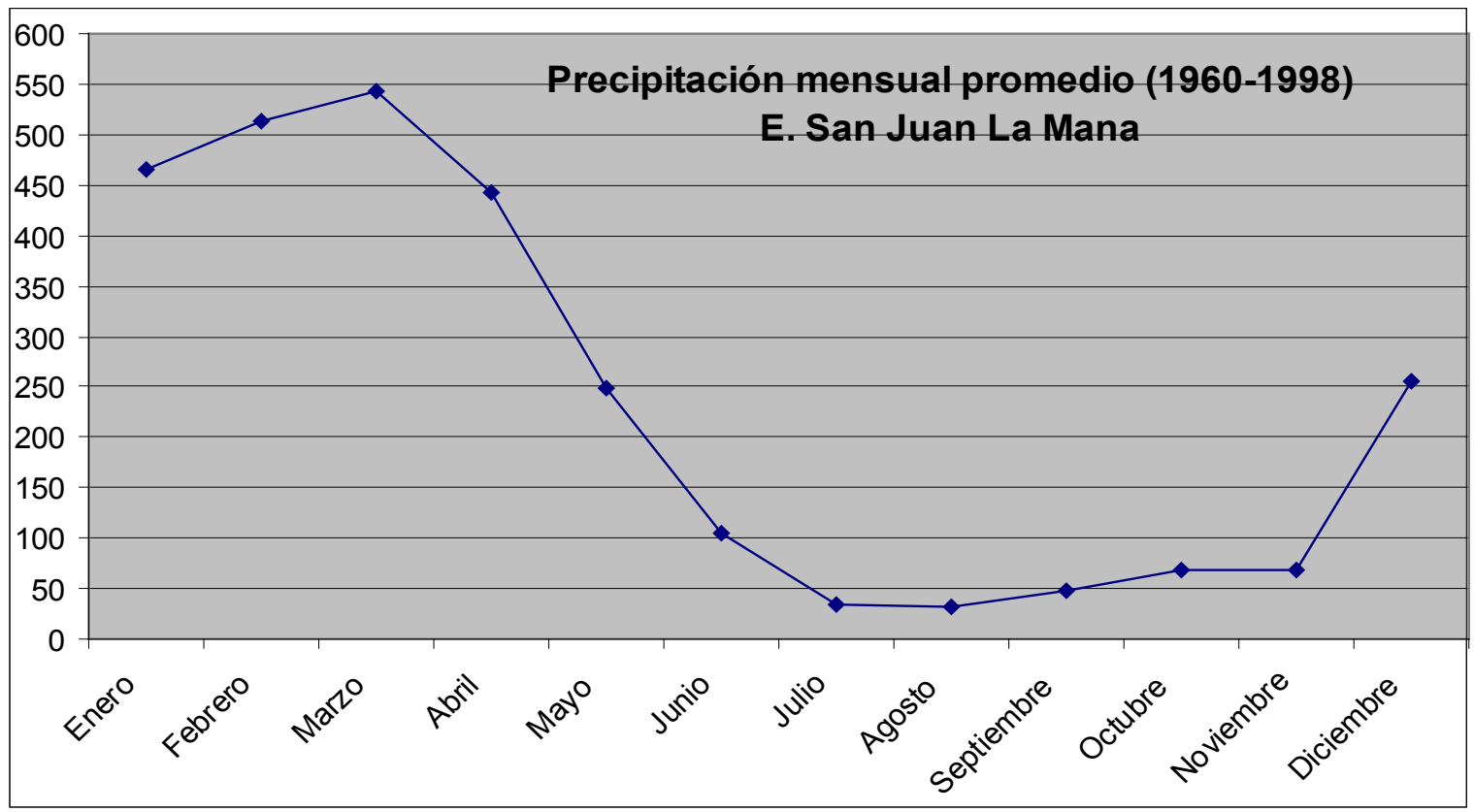

Figura 5. Precipitación mensual promedio (1960 - 1998) Estación San Juan La Mana INAMHI.

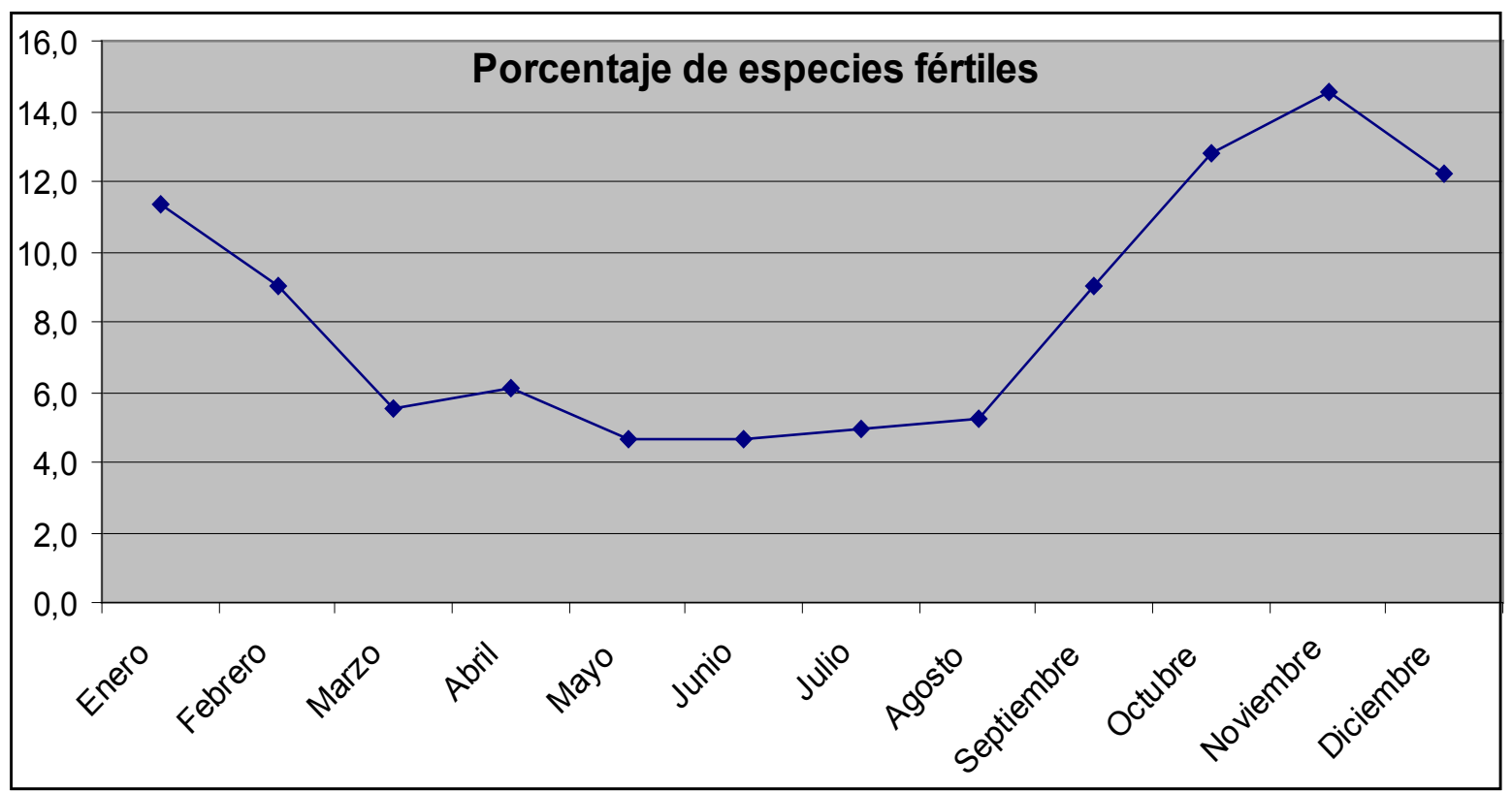

Figura 6. Porcentaje de especies fértiles en la Estación 'Sacha Wiwa'. 


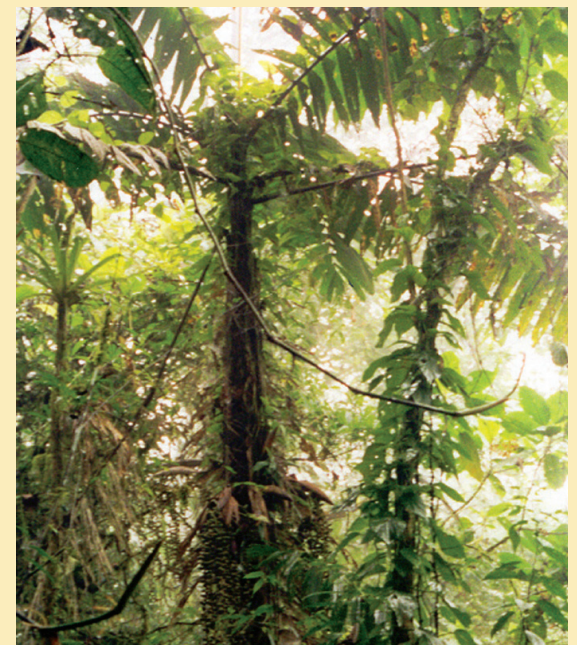

Wettinia equalis (O.F.Cook \&

Doyle)R.Bernal ARECACEAE

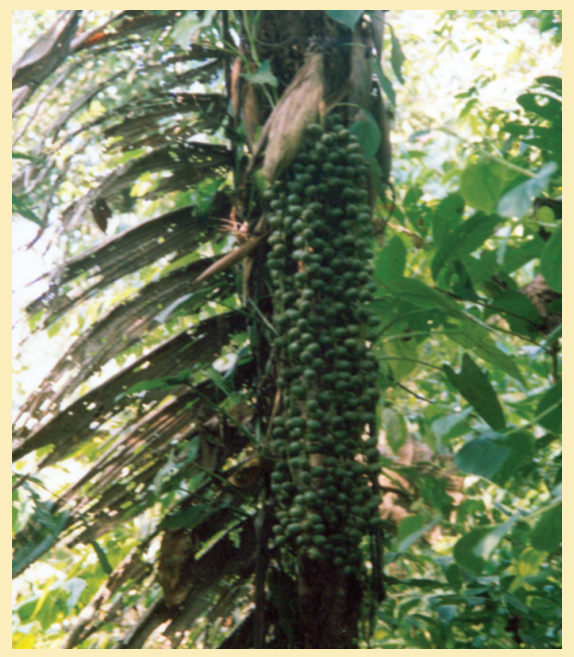

Wettinia equalis (O.F.Cook \& Doyle) R.Bernal ARECACEAE

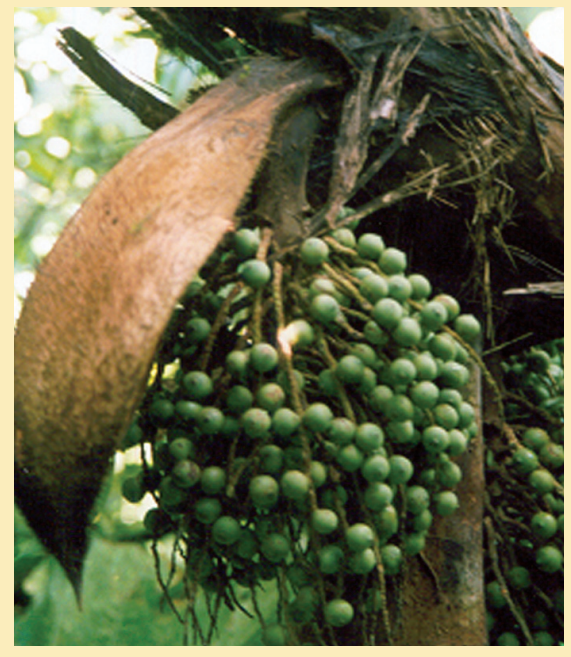

Bactris setulosa $\mathrm{H}$. Karst. ARECACEAE

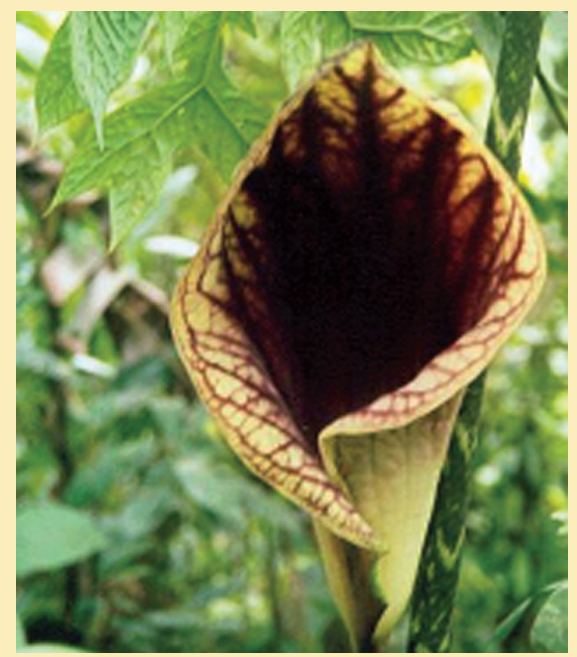

Dracontiun croatii G.H. Zhu ARACEAE

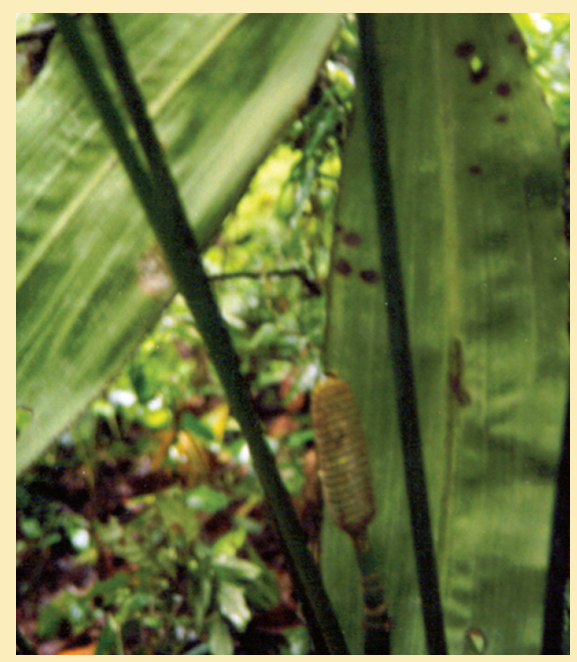

Cyclanthus bipartitus Poit. CYCLANTHACEAE

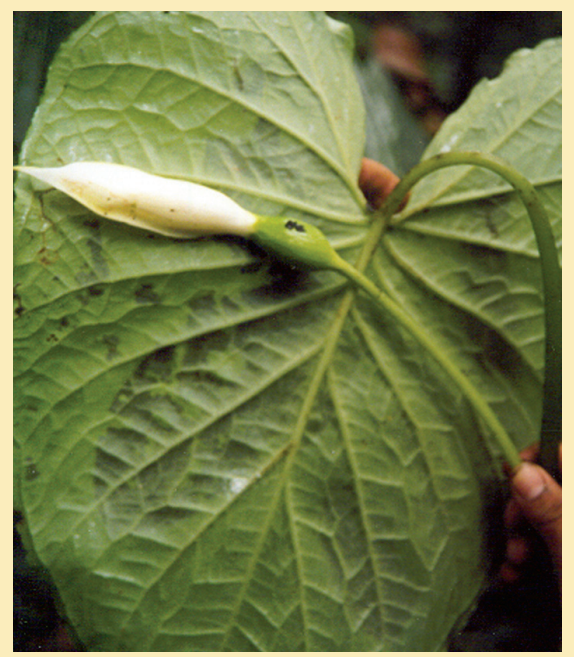

Xanthosoma eggersii Engl ARACEAE

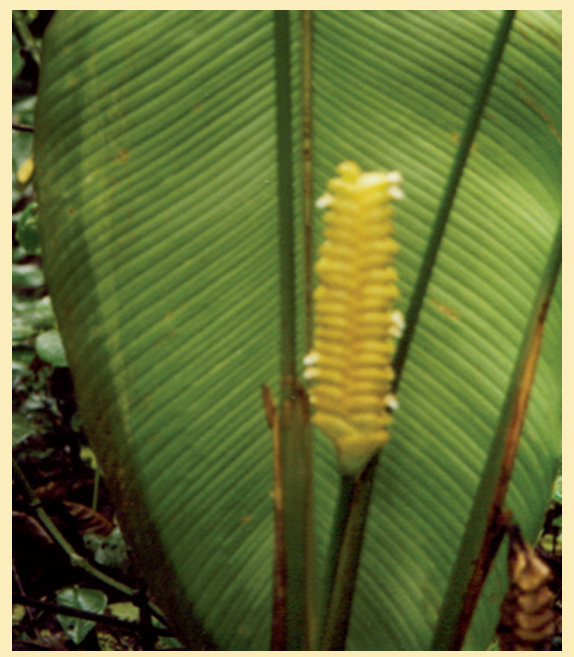

Calathea crotalifera Watson MARANTACEAE

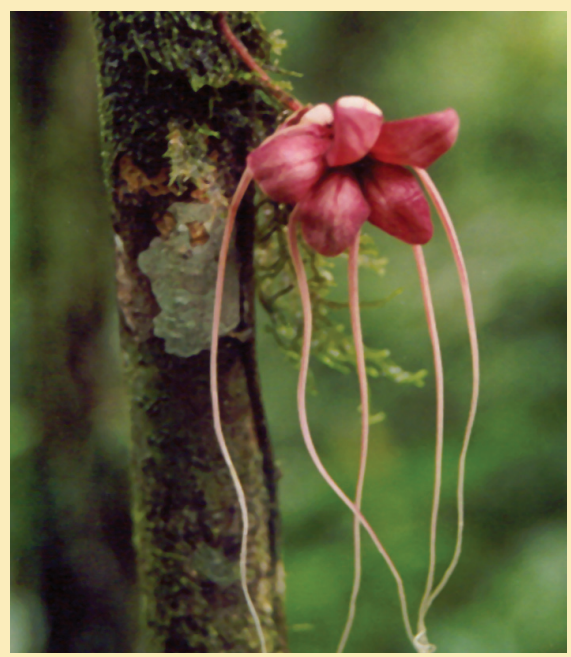

Herrania balaensis P.Preuss STERCULIACEAE

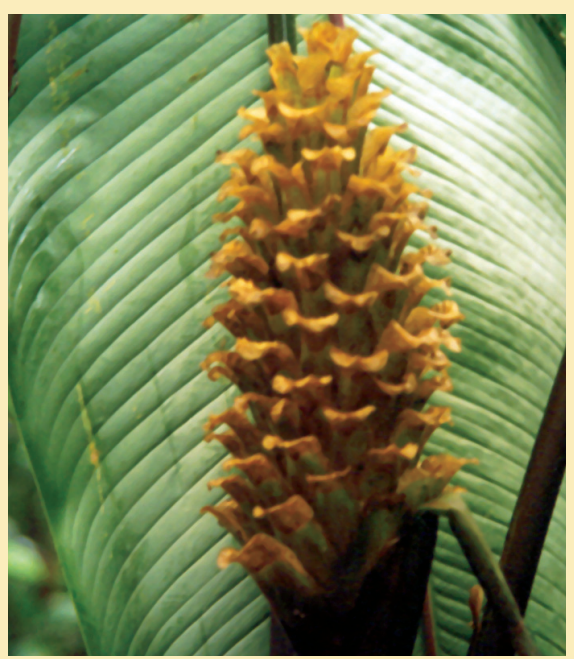

Costus guanaiensis Rugby MARANTACEAE

Figura 7. Especies de la Estación 'Sacha Wiwa'. 


\section{Conclusiones y recomendaciones}

Los taxones más importantes en la estructura del bosque son: Wettinia equalis, Miconia brachycalyx y Aegiphyla alba; Las familias más importantes en la estructura del bosque son Arecaceae y Melastomataceae.

Los bosques del lugar demuestran poca importancia para la explotación maderera. Pero es de interés en el campo de la investigación y del turismo ya que es un refugio de la flora y la fauna. Se recomienda incluir en el plan de manejo de la estación la introducción de especies nativas con valor comercial que alternen con las especies dominantes en la estructura del bosque.

El Bosque de la Estación 'Sacha Wiwa' tiene un índice de diversidad calculado de 7,63; lo que nos indica que el bosque se ubica en el nivel de diversidad baja. Esto se debe a que se trata de un bosque secundario que se encuentra en recuperación y está poblado en su mayoría por especies de ciclos de vida cortos como hierbas, arbustos, trepadoras y epíitas. Este bosque se encuentra en recuperación y tiene una baja diversidad en especie forestales, pero son especies hospederas de epifitas.

En este lugar existen 20 especies endémicas de las cuales 2 están en la categoría 'En Peligro'; 2 en la categoría 'Vulnerables' y 2 están en la categoría 'Casi Amenazadas'. Este número de especies endémicas nos permite establecer prioridades para la conservación de la zona e incrementar los estudios de la flora del lugar.

Un dato interesante para la flora de la Estación 'Sacha Wiwa' es la presencia de dos especies nuevas para el lugar, las especies están por publicarse y tendrían el nombre Anthurium sachawiwense y Anthurium pucayacuense; Esto demuestra la necesidad de ampliar los estudios botánicos en la zona; ya que es un lugar interesante en la diversificación de la flora especialmente a nivel de la epifitas. Los futuros estudios de la flora del lugar deben planificarse para el periodo entre los meses de octubre y enero. Es decir en la época de mayor fertilidad de la flora.

\section{Referencias bibliográficas}

Brenes, G. I99I. Parcelas de muestreo permanente, una herramienta de investigación de nuestros bosques. Programa de Restauración y Silvicultura del Bosque seco A.C.G. Guanacaste. Costa Rica.

Bonilla,A. 1983. Recursos Forestales y Zonas Protectoras. Editorial Tecnológica de Costa Rica.

Cerón, C. 1993. Manual de Botánica Ecuatoriana.
Escuela de Biología y Química. F.F.L y C.E. Universidad Central del Ecuador. Quito.

Cerón, C,W. Palacios, R. Valencia y R. Sierra 1999. Las Formaciones Naturales de la Costa del Ecuador. Pág. 55-77 En: R. Sierra (Ed.) Propuesta preliminar de un sistema de clasificación de vegetación para el Ecuador continental. Proyecto INEFAN/GEF-BIRF y EcoCiencia, Quito.

Encarta, 2007. Enciclopedia Encarta ${ }^{\circledR} 2007$ [DVD]. (C) 1993-2006 Microsoft Corporation.

INAMHI, 1998. Series de Datos Metereológicos; Estación San Juan La Mana. 223msnm periodo 1960 - 1998. Instituto Nacional de Meteorología e Hidrología. Ministerio de Energía y Minas, Quito-

Jørgensen, P.M. \& S. León-Yánez. 1999. Catalogue of the Vascular Plants of Ecuador. Missouri Botanical Garden Press.

Larrea, M. 1997. Respuestas de las epifitas vasculares a diferentes formas de manejo del bosque nublado, Bosque protegido Sierra Azul, Zona de Amortiguamiento de la Reserva Ecológica Cayambe - Coca, Napo - Ecuador.

Leaño, C. y P. Saravia. 1998. Monitoreo de parcelas permanentes de medición en el bosque Caimanes. Proyecto de Manejo Forestal Sostenible BOLFOR. USAID. Bolivia.

Palacios, W., G. Tipaz y C. Aulestia 1997. Inventarios florísticos y análisis vegetacionales en la parte baja del noroccidente del Ecuador 1992-1994. pág: 36I-374. En: Mena P. A. Soldi, R.Alarcón, C. Chiriboga y L. Suárez (eds.). Estudios Biológicos para la Conservación, Diversidad, Ecología y Ednobiología. Ecociencia. Quito.

Uslar, Y., B. Mostacedo y M. Saldias 2003. Composición, Estructura y Dinamica de un Bosque seco semideciduo en Santa Cruz, Bolivia. Proyecto de Manejo Forestal Sostenible BOLFOR. USAID. Bolivia.

MOBOT. Trópicos. Disponible en línea en: <www.mobot.org>. Consulta 30 de septiembre de 2010.

Valencia, R. 2000. Libro rojo de las plantas endémicas vasculares. Herbario QCA.PUCE. 